\title{
Etnobotánica y fitoquímica de Selaginella willdenowii (carpintero) en algunas comunidades del Chocó biogeográfico
}

\section{Ethnobotany and phytochemistry of Selaginella willdenowii (carpintero) in some communities at biogeography Chocó}

\author{
Betsabe Caicedo-Chaverra ${ }^{1}$, Yohana Mendoza-Valencia ${ }^{1}$, \\ Julia del Carmen Palacios-Lloreda ${ }^{2}$
}

\begin{abstract}
Resumen
Para conocer el uso que las comunidades de Quibdó, Tutunendo y Guayabal dan a Selaginella willdenowii (carpintero) y la presencia de potenciales metabolitos secundarios en esta planta, se condujo un estudio etnobotánico y fitoquímico sobre esta especie. La información etnobotánica se obtuvo mediante la realización de encuestas semiestructuradas a la población de las comunidades analizadas. El análisis fitoquímico incluyó una marcha fitoquímica cualitativa y análisis cromatográficos que se desarrollaron en la Universidad de Antioquia con el apoyo del Grupo de Investigación en Química de Plantas Colombianas. Nuestros resultados a nivel etnobotánico mostraron que el carpintero presenta tres categorías de uso en las comunidades encuestadas: medicinal, mágico-religioso y ornamental. A nivel fitoquímico, se observó que esta especie presenta una mayor concentración de triterpenoides y esteroides, seguidos por flavonoides, y en menor cantidad de alcaloides. La presencia de estos compuestos en S. willdenowii sugieren facultades antimicrobianas, anticancerígenas, cardiotónicas y analgésicas que se asociaron con el uso medicinal que las comunidades estudiadas dan a esta especie.
\end{abstract}

Palabras clave: Etnobotánica y fitoquímica; Selaginella willdenowii; Carpintero; Quibdó; Tutunendo; Guayabal.

\begin{abstract}
In order to know the type of uses that human communities of Quibdó, Guayabal Tutunendo give to Selaginella willdenowii (carpintero), as well as to determine the secondary metabolites present in this plant we conducted anethnobotanical and a phytochemical study. Etnobotanical information was obtained through semistructured interviews among analyzed human communities. Phytochemical analyses include a qualitative phytochemical march and chromatographic analyses conducted at the University of Antioquia, with the support of the Research Group on Plant Chemistry of Colombia). From an ethnobotanical standpoint, our results showed that the carpintero has three categories of use in the surveyed human communities: medicinal, magical-religious and ornamental. Our phytochemical results showed that this plant species has higher concentrations of triterpenoids and steroids, followed by flavonoids, and a lesser amount of alkaloids. The presence of these compounds in S. willdenowii suggests antimicrobial anticancer, cardiotonic, analgesic qualities we associate with the medicinal use that human communities give to this species in the study area.
\end{abstract}

Keywords: Ethnobotany and phytochemistry; Selaginella willdenowii; Carpenter; Quibdó; Tutunendo; Guayabal.

1 Grupo de Investigación en Bioprospección Diversidad y Cultura, Universidad Tecnológica del Chocó, Quibdó. Colombia. e-mail: betcacha@yahoo.es yohanitamen@yahoo.es

2. Grupo de Investigación de la Flora Chocoana. Docente, Programa de Biología con Énfasis en Recursos Naturales, Universidad Tecnológica del Chocó, Quibdó, Colombia.e-mail: jcpl173@hotmail.com

Fecha recepción: Enero 18, $2011 \quad$ Fecha aprobación: Marzo 2, 2011

() Rev. Biodivers. Neotrop. 2011; 1 (1): 55-64 


\section{Introducción}

El departamento del Chocó goza de gran variedad de plantas que son aprovechadas por sus pobladores como medicinales, ornamentales y mágico-religiosas. Por esta razón, resulta importante estudiar la etnobotánica y la composición química de aquellas especies que utilizan de manera tradicional las comunidades negras e indígenas de esta región. Se destacan entre éstas las Pteridofitas, plantas vasculares que agrupan a los helechos y plantas afines.

En el Chocó biogeográfico se registran 425 especies de Pteridofitas, concentrándose el mayor número en el departamento del Chocó con 364 (Murillo, 2004), lo que demuestra la diversidad y abundancia de algunas familias, entre ellas la Selaginellaceae con su único representante Selaginella. Este género cuenta con alrededor de 750 especies distribuidas principalmente en zonas tropicales, crecen en lugares cálidos y húmedos, aunque existen algunas especies en desiertos y zonas de alta montaña (Prada, 2004). El Chocó biogeográfico cuenta con cerca de 38 especies de las cuales 24 están en el departamento del Chocó (Forero y Gentry, 1989; Murillo, 2004).

Las especies del género Selaginella se caracterizan por ser plantas terrestres, rupícolas y en ocasiones epifitas, tallos articulados o no articulados, raíces en su mayoría adventicias y dicótomas, localizadas en los extremos de los rizóforos originados en la superficie ventral o dorsal de las bifurcaciones del tallo; hojas isofilas arregladas en cuatro hileras, dos en la superficie dorsal y dos laterales; estróbilos al final de las ramas terminales (Alston, 1955; Alston et al., 1981; Gregory et al., 1979; Jermy, 1986; Valdéspino, 1993).

El carpintero (Selaginella willdenowii), es originario del Viejo Mundo, predominante de los países Myanmar, Malasia, Indonesia y Filipinas e introducido al Nuevo Mundo incluyendo países como Estados Unidos, Panamá, Colombia (Antioquia, Chocó), Jamaica y Puerto Rico, en bosques húmedos, bordes de camino, desde los 50-900 msnm. Es una planta terrestre con tallos rojizos a verdes o pajizos, erectos, glabros, isofilos desde la base hasta la primera ramificación; hojas axilares auriculadas, rojizas por el envés y por el haz verdosas (Alston, 1955; Alston et al., 1981; Gregory et al., 1979; Jermy, 1986; Valdéspino, 1993).
A los miembros de la familia Selaginellaceae se les atribuyen potencialidades medicinales (Forero, 1980); pruebas fitoquímicas realizadas por Gallathri (2005) reportan propiedades inmunomudulatorias, antioxidantes; sin embargo, otras investigaciones realizadas en China por Ling-Yun Ma et al. (2003) describen potencialidades antivirales. Shun $\mathrm{Fa}$ et al. (2007) a través de algunas investigaciones sobre el género Selaginellas, determinó sus propiedades antimetástasis y Chang-Ming Sun et al. (1997) al realizar observaciones a biflavonoides presentes en especies de este género determinó su actividad citotóxica. En este sentido, algunos estudios fitoquímicos del género Selaginella reportan aislamiento de cromonas (Ling-Yun Ma, 2003), biflavonoides (Chan-Woo Lee et al., 2008), sesquilignanos (Yan Zhi Wang, 2007) benzofuranos (Xiao Ke Zheng et al., 2008) y glicoalcaloides (Ling-Yun Ma et al., (2003).

En el departamento del Chocó se han registrado muy pocos aportes sobre estudios realizados con el carpintero. Se destacan los aportes de Mosquera (1996) que incluye un listado de las plantas más utilizadas para combatir algunas enfermedades donde se determinó que esta especie se utiliza para eliminar los áscaris. De igual manera, Palacios (2008) verificó la distribución geográfica de la especie en la localidad y comentó aspectos puntuales sobre su hábitat, demostrando que crece en lugares húmedos y sombreados, donde los sustratos más aptos para su crecimiento son la hojarasca y el pantano, aquí se registró el mayor porcentaje de especies y en menor proporción la materia orgánica viva (epifitas).

Las anteriores evidencias, nos muestran que el conocimiento que se tiene de la especie Selaginella willdenowii «carpintero» en el departamento del Chocó, en especial sobre sus potencialidades fitoquímicas, genéticas y etnobotánicas, entre otras, es pobre, debido a que muchas de las investigaciones realizadas se orientan hacia el conocimiento taxonómico y distribución de las especies dejando de lado otros atributos que pueden permitir un mayor aporte a las ciencias biológicas.

Por esta razón, el propósito de esta investigación es cuantificar el uso etnobotánico que las comunidades de Quibdó, Guayabal y Tutunendo han dado a esta especie y generar un conocimiento científico a través de la determinación cualitativa de algunos 

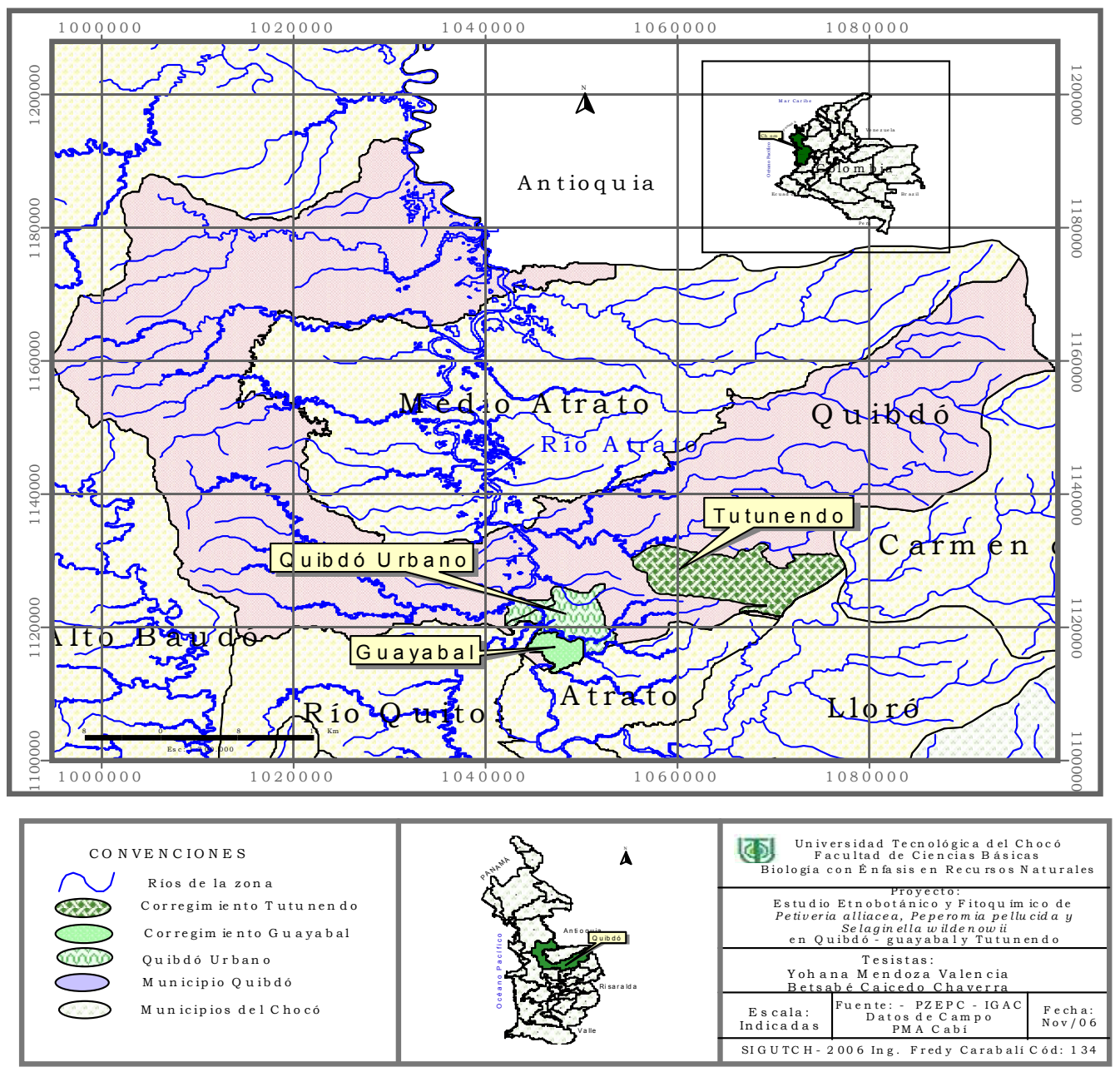

Figura 1. Ubicación de las áreas de muestreo S. willdenowii en el municipio de Quibdó, Chocó.

metabolitos secundarios que presenta y que pueden aportar a la medicina alternativa en dichas comunidades.

\section{Materiales y métodos}

Área de estudio. El levantamiento de la información etnobotánica se llevó a cabo en la cabecera municipal de Quibdó (barrios de la zona norte: El Futuro, Villa España, La Victoria, La Gloria, Samper) y los corregimientos de Tutunendo y Guayabal (Figura 1).

El municipio de Quibdó, limita al norte con el municipio del Medio Atrato, al sur con los municipios de Río Quito y Lloró, al oriente con el municipio del Carmen de Atrato, al nororiente con el departamento de Antioquia y al occidente con el municipio de Alto Baudó; su cabecera municipal, que lleva su mismo nombre, se encuentra entre 43 y $53 \mathrm{msnm}$ y tiene una temperatura promedio de $28^{\circ} \mathrm{C}$, según el Plan de Ordenación Territorial de Quibdó (POT Quibdó, 2003). Está ubicado en la región de calmas ecuatoriales y según el sistema de Holdrige (1963) se encuentra entre las zonas de vida de bosque muy húmedo tropical (bmh-T) y bosque pluvial tropical (bp$\mathrm{T})$, que se caracterizan por altas precipitaciones y temperaturas superiores a $24^{\circ} \mathrm{C}$. Tiene un área de $3337.5 \mathrm{~km}^{2}$ y una población de 97,714 habitantes, que representa el 32\% del total del departamento. El 65\% se encuentra en el área urbana. En las zonas de la cuenca del Atrato y sus afluentes las principales actividades económicas son la pesca complementada con la agricultura; subiendo los afluentes, la agricultura aumenta, encontrándose que las áreas de las quebradas aptas para esta actividad son reducidas. Al subir por los afluentes la combinación de la pesca y 
la agricultura pasa a ser agro-minera y minería como actividad principal. Es de resaltar que en las poblaciones del Atrato la pesca está afectada por causa de los desechos industriales y domésticos de Quibdó (POT Quibdó, 2003) (Figura 1).

El corregimiento de Guayabal, está localizado al norte de la ciudad de Quibdó, a unos $15 \mathrm{~km}$ vía carreteable. Limita al norte con el corregimiento de San Antonio de Ichó, al oriente con el corregimiento de La Troje, al occidente con la vereda el Barranco y al sur con el área urbana de Quibdó. Tiene una población de 400 habitantes, distribuida en una superficie de 5.14 ha. La caracterización del tipo de subsistema productivo realizado por la comunidad de Guayabal muestra un sistema minero-agrícola, y se identifica como actividad principal la minería, que es de tipo artesanal y semi industrial, los equipos utilizados son motobombas y dragas. Como actividad complementaria se práctica la agricultura. Astronómicamente se encuentra a $\operatorname{los} 5^{\circ} 44^{\prime} 00^{\prime \prime}$ al norte y $76^{\circ} 38^{\prime} 45^{\prime \prime}$ al oeste, presenta una altura de $48 \mathrm{msnm}$; con respecto a la precipitación es una zona de pluviosidad moderadamente alta (5909 $\mathrm{mm}$ a $8494 \mathrm{~mm})$, en términos de temperatura pertenece a una zona megatermal $\left(25.7^{\circ} \mathrm{C}\right.$ a $\left.27.9^{\circ} \mathrm{C}\right)$, la humedad va de perhúmedo a superhúmedo (248.9 msnm a $385.8 \mathrm{msnm}$ ) (Rangel y Arellano, 2004) (Figura 1).

El corregimiento de Tutunendo, se encuentra ubicado al oriente de la capital del departamento del Chocó, Quibdó, a 19 km sobre la carretera que conduce a la ciudad de Medellín. Según el Plan de Ordenación Territorial de Quibdó (POT Quibdó, 2003), cuenta con cerca de 3500 habitantes distribuidos en 472 viviendas; es uno de los corregimientos más importantes del municipio de Quibdó por su diversidad biológica y cultural, aunque presenta un alto índice de necesidades básicas insatisfechas. La caracterización del tipo de subsistema productivo realizado por el Consejo Comunitario Local de la comunidad de Tutunendo muestra un sistema agrícola-mineromaderero, y se identifica como actividad principal la agrícola y pecuaria. Los principales productos que se comercializan son: plátano (Musa paradisiaca), arroz (Orazi sativa), maíz (Zea maíz), cría de especies menores como cerdo y aves, actividades que son consideradas por la comunidad como una fuente de ingresos; la mayor dificultad que presenta la comunidad es el mercadeo, transporte y falta de crédi- to. Como actividad complementaria, tienen la minería y la explotación de madera, esta última se da sin ninguna licencia de aprovechamiento. Las especies que más se comercializan son: abarco (Cariniana pyriformis), cedro (Cedrela odorata), roble (Tabebuia rosea) (Mahecha, 1997); dentro de las actividades que realizan de manera ocasional se encuentran la caza de especies menores y la pesca; sin embargo, el recurso más importante es el agua y su densa vegetación (POT Quibdó, 2003). Presenta zona de vida bosque pluvial tropical (bp-T), su altitud es de $90 \mathrm{msnm}$ y presenta una precipitación media anual de 11394 mm de tiempo bimodal (Gentry, 1986).

Métodos. Para el conocimiento etnobotánico, se realizaron encuestas semiestructuradas siguiendo la metodología propuesta por Adu Tutu et al. (1979) que permitió determinar las categorías de uso de la especie y evaluar el impacto socioeconómico y cultural, teniendo en cuenta las creencias y tradiciones de los pobladores de los sitios estudiados. Los encuestados se eligieron entre personas mayores de 60 años de cada comunidad, porque ellos por su mayoría de edad, poseen experiencias y conocimientos que han adquirido de generación en generación. También se encuestaron curanderos y parteras, quienes por su oficio, en la mayoría de los casos utilizan plantas tanto medicinales como mágico-religiosas, al igual que madres cabeza de hogar y otras personas mayores de 25 años que pudieran tener algún conocimiento acerca de las plantas, en especial del carpintero.

La determinación de los metabólicos secundarios, se llevó a cabo en la Universidad de Antioquia, Facultad de Ciencias Exactas y Naturales del Instituto de Química, siguiendo la metodología propuesta por Saez y Noreña (2008), miembros del grupo de química de plantas colombianas. Se tomaron 100 gramos del material vegetal seco al ambiente y molido utilizando toda la planta, se extrajeron en un percolador con 1 litro de etanol. La solución etanólica se evaporó al vacío en un rotaevaporador a un volumen de 40 $\mathrm{ml}$, luego se adicionaron $50 \mathrm{ml}$ de agua destilada y se realizaron particiones en un embudo de decantación con $100 \mathrm{ml}$ de éter de petróleo, $100 \mathrm{ml}$ de diclorometano y $100 \mathrm{ml}$ de acetato de etilo, obteniéndose de esta manera tres soluciones. Las soluciones orgánicas se secaron con sulfato de sodio anhidro para eliminar el agua remanente, se filtraron y se concentraron a sequedad (Figura 2). 


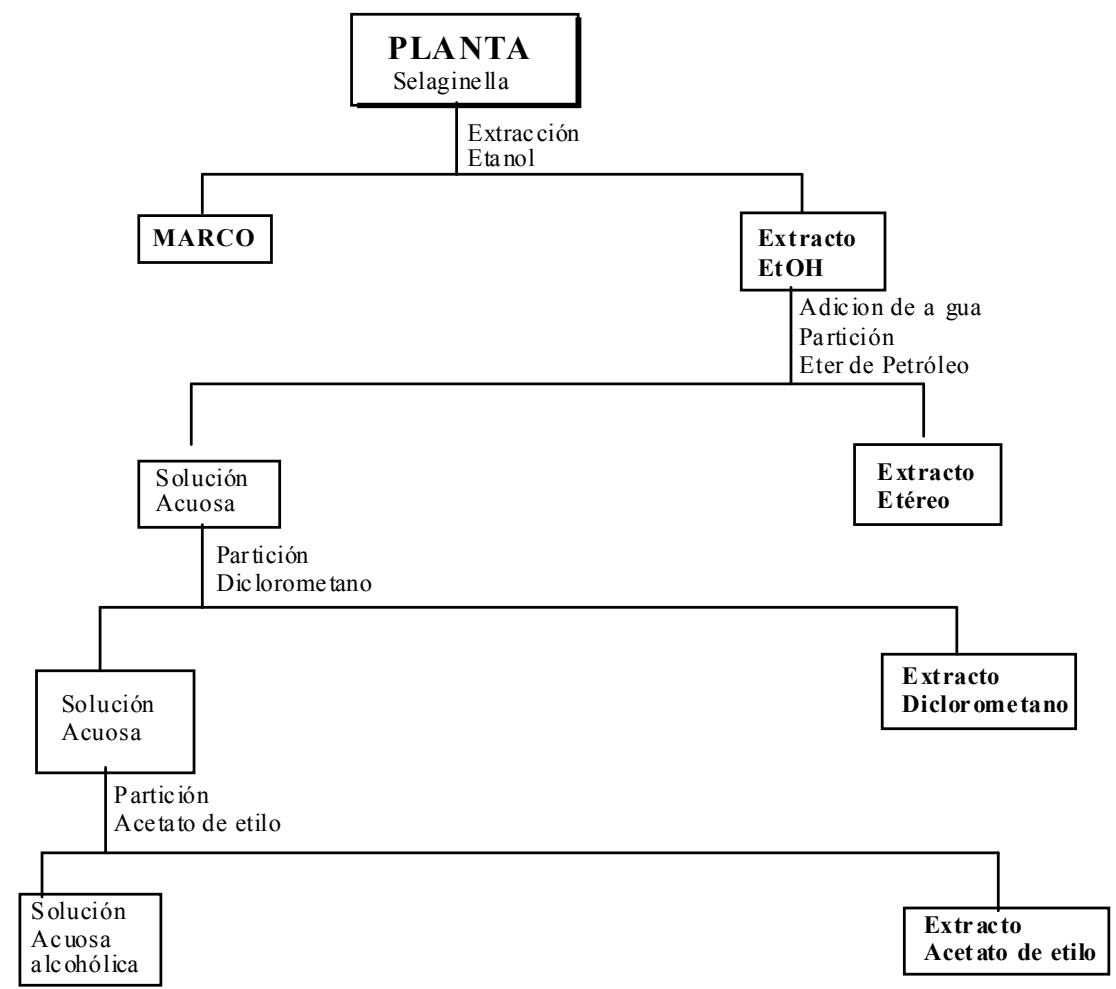

Figura 2. Procedimiento para la extracción de los metabolitos secundarios en S. willdenowii .

Tabla 1. Pruebas fitoquímicas realizadas a la especie S. willdenowii

\begin{tabular}{ll}
\hline \multicolumn{1}{c}{ Metabolitos } & \multicolumn{1}{c}{ Pruebas } \\
\hline Compuestos fenólicos & Cloruro férrico \\
Táninos & Prueba con gelatina-sal \\
Flavonoides & Shinoda \\
Triterpenoides & Liebermann-Bourchard \\
Esteroides & Liebermann- Bourchard \\
Quinonas & Bortraeger \\
Cardiotónicos & Ensayo de Kedde \\
Alcaloides & Dragendorff, Mayer, Valser \\
& y Reineckato de amonio \\
\hline
\end{tabular}

La marcha fitoquímica se llevó a cabo en el extracto etanólico bruto y también sobre los extractos de éter de petróleo, diclorometano y acetato de etilo. Se realizaron pruebas para los siguientes metabolitos: fenoles y taninos con cloruro férrico, flavonoides con la prueba de Shinoda o cianidina, triterpenoides y esteroides con la prueba de Liebermann-Bourchard, alcaloides con las reacciones de Dragendorff, Mayer, Valser y Reineckato de amonio, quinonas con la reacción de Bortraeger y la prueba de cardiotónicos con la reacción de Kedde (Tabla 1).
La información que se obtuvo se analizó mediante el cálculo de los índices cuantitativos para la especie S. willdenowii (carpintero), los que se utilizaron como indicadores del grado de consenso de informantes propuesto por Adu-Tutu, Elvin-Lewis (1980), determinando el uso y la importancia cultural de la especie en las comunidades investigadas.

\section{Resultados y discusión}

Se encuestaron 584 personas, entre curanderos, parteras, madres cabeza de hogar y mayores de 25 años, observándose que el $57.4 \%$ de la población (335 personas) conocen la especie, porque la han utilizado, cultivan o venden (Tabla 2). Estos resultados se pueden deber a que estas personas en primera instancia provienen de lugares en donde le dan mayor credibilidad a las plantas medicinales y en segunda instancia, por su situación económica, se les dificulta acceder a los médicos especialistas; a pesar de ello hay un alto porcentaje de la población que manifiesta desconocimiento de esta especie (42.6\%: 249 personas). La información que aportaron los entrevistados de Tutunendo, Guayabal y Quibdó permitió clasi- 
Tabla 2. Lista de personas encuestadas teniendo en cuenta su conocimiento sobre la especie en las zonas de estudio

\begin{tabular}{|c|c|c|c|c|c|}
\hline \multirow{2}{*}{ Zonas } & \multicolumn{4}{|c|}{ Personas encuestadas $u$ oficios } & \multirow[b]{2}{*}{$\begin{array}{l}\text { Subtotal } \\
\text { encuestado }\end{array}$} \\
\hline & Curanderos & Parteras & $\begin{array}{l}\text { Madres cabeza } \\
\text { de hogar }\end{array}$ & $\begin{array}{c}\text { Personas mayores } \\
\text { de } 25 \text { años }\end{array}$ & \\
\hline Quibdó & 6 & 4 & 202 & 184 & 396 \\
\hline Tutunendo & 11 & 1 & 31 & 73 & 116 \\
\hline Guayabal & 2 & 1 & 13 & 56 & 72 \\
\hline Total & 19 & 6 & 246 & 213 & 584 \\
\hline
\end{tabular}

ficar a la especie en tres categorías de uso: medicinal (54\%), mágico-religiosa (39\%), y ornamental (7\%), esta última categoría la utilizan para adornar las viviendas.

La información que se obtuvo en esta investigación se relaciona con lo encontrado por Cardona (1994), quien en su listado de plantas medicinales del Alto Baudó, Chocó, manifiesta que el carpintero sirve para curar dolores de muelas; por su parte Escobar et al. (2003) en su investigación sobre etnobotánica y comercialización de plantas aromáticas y medicinales en Quibdó registra que el carpintero se utiliza para sacar la frialdad del cuerpo. En este mismo sentido Moreno y Zapata (2008), en su trabajo flora y fauna de uso medicinal en el municipio de Cértegui al igual que Perechala et al. (1995), en su estudio sobre el conocimiento tradicional en la costa pacífica colombiana, sostienen que el carpintero acelera las contracciones uterinas o dolores de parto y sirve para destramar, utilizando las hojas en bebedizos y baños.

De igual manera Mosquera (1996), determinó que esta especie se utiliza para eliminar los áscaris, mientras que Mosquera y Rentería (2005) realizaron una caracterización sobre huertos caseros en Pacurita y reportan a esta especie como medicinal, sin especificar que enfermedades cura.

En cuanto al uso mágico-religioso, la comunidad usa la especie para proteger las viviendas, enamorar y curar enfermedades, combinándola con otras plantas como albahaca (Ocimum bacilicum), amansa justicia (Justicia pectoralis), quereme, (Peperomia pernambucensis), hierbabuena (Menta sativa) y con otros productos como panela chocoana o miel de abejas, colonias, siete esencias, narcisos negros y menticol, que proporcionan aroma al preparado, utilizando el secreto del carpintero para obtener mayor efectividad. Estos productos los preparan en formas de perfumes, baños, balsámicas y riegos siguiendo algunos rituales, para lo cual tienen en cuenta el tiempo (fechas y horas) especial para que cause el efecto esperado, dependiendo del requerimiento (enamorar, proteger la vivienda y el cuerpo, y sacar maleficios). De esta manera, clasifican las enfermedades en frías (cólicos menstruales, entuertos, pasmo, frialdad en el acto sexual bien sea en el hombre o mujer, resfriados, entre otras) y calientes (enfermedades de los riñones, reumatismo, etc.).

La clasificación anterior es corroborada por Perechala et al. (1995), cuando registra que las enfermedades se clasifican en frías, cuando los quebrantos de salud aparecen de la cintura hacia la parte inferior del cuerpo, siendo éstas más frecuentes en las mujeres. Ejemplo de dichas enfermedades son las relacionadas con la sangre, la matriz y derivadas del parto. Mientras que las enfermedades calientes van de la cabeza a la cintura; algunas de ellas son la retención urinaria, reumatismo, bazo y el hígado, entre otras. En este mismo sentido, Echavarría (1999) menciona que cuando se habla de enfermedad hay que tener en cuenta tres factores: la etiología o causa de la enfermedad (si es por naturaleza: fría o caliente); la nosología, lectura u observaciones de indicios o síntomas y la terapéutica o modos de curación (acciones, baños, bebedizos o con plantas, secretos o maniobras).

Con base en la anterior afirmación, el investigador sostiene que los males son divinos (cuando la enfermedad proviene de Dios) y malignos (si es de otro tipo); así, Caicedo (1983) sostiene que las oraciones se rezan a los santos, al diablo y otras fuerzas sobrenaturales para la invocación de protección diaria; de igual manera, Perechala et al. (1995) manifestaron que la magia se utiliza para provocar algunas enfermedades o males 
(la trama de las parturientas, dolores de cabeza intempestivos y la muertes repentinas), pero también se utiliza para la curación y si no se obtiene beneficio con las plantas, se recurre al canto de la chicha o jai; asimismo, se usan especies vegetales para obtener suerte en los juegos de azar.

En los saberes tradicionales de los chocoanos el uso mágico-religioso, juega un papel muy importante, porque a través de él se manipula a la naturaleza con fuerzas maléficas o benéficas concentradas en la mente, de allí la creencia en secretos u oraciones, lo que produce un tipo de alteración en el equilibrio de las personas que resulta inexplicable para la ciencia, pero no por ello se deben dejar pasar desapercibidos o restarles importancia, porque por medio de dichos «secretos u oraciones» se le pide a las plantas, a Dios o a los santos, para que ayuden e intervengan en la curación de enfermedades, enamorar o hechicería.

En este estudio se encontró que el $10 \%$ de la población (58.4 personas) que ha utilizado la especie, lo hacen con el «secreto del carpintero», que guardan con recelo, como información confidencial; ellos expresan que este actúa directamente en los beneficios que se quieren obtener al utilizar la planta, resaltando así las creencias religiosas que tienen las comunidades negras. En este sentido Bueno y Palacios (2006), en una investigación sobre las especies útiles para la preparación de balsámicas en los municipio de Opogodó y Novita, registraron que el carpintero se usa para protección del cuerpo, porque la especie evita el alojamiento de maleficios; de igual forma Perechala et al. (1995) en su estudio sobre la recuperación del pensamiento mágico de las comunidades afrochocoanas, resaltan la importancia de la magia en la curación de algunas enfermedades y maleficios; además hacen seguimiento a curanderos, brujos y otras personas poseedoras de información sobre prácticas mágico-religiosas en las enfermedades y sus curas, los secretos u oraciones, mitos y leyendas entre los que está la leyenda de la hierba del carpintero. Por otra parte, Mosquera y Rentería (2005), en su caracterización de huertos caseros en Pacurita, reportan el uso mágico-religioso de esta especie pero sin especificar su acción.

A nivel socioeconómico los resultados de la encuesta muestran que el $60 \%$ de la población encuestada (350.4 personas) compra el carpintero a un valor que oscila entre 1000 y 5000 pesos el mano- jo y el $40 \%$ restante ( 233.6 personas), vende la especie para beneficio propio, lo que, sirve de aporte al sostenimiento diario junto con lo que reciben por la venta de sus otros productos, reflejando el impacto socioeconómico y cultural de la especie en las comunidades.

En el análisis fitoquímico preliminar de la especie $S$. willdenowii se obtuvieron tres soluciones, $200 \mathrm{mg}$ de extracto etéreo, $320 \mathrm{mg}$ de extracto de diclorometano y $125 \mathrm{mg}$ de extracto de acetato de etilo; luego se realizaron pruebas para los siguientes metabolitos: fenoles con cloruro férrico y taninos con la prueba de gelatina-sal, en las que no se formó precipitado y se consideró la prueba negativa; flavonoides con la prueba de Shinoda o cianidina, donde se observó la aparición de coloraciones naranja a violeta por lo que se consideró positiva; triterpenoides y esteroides con la prueba de Liebermann-Bourchard, se observaron cambios de coloración y se consideró positiva; alcaloides con las reacciones de Dragendorff, Mayer, Valser y Reineckato de amonio, se considera positiva porque aparecen precipitados en la mayoría de los tubos; quinonas con la reacción de Bortraeger, se consideró negativa y la prueba de cardiotónicos con la reacción de Kedde considerada negativa porque no hubo presencia de coloración púrpura o violácea (Tabla 3 ).

En el análisis cromatográfico de los diferentes extractos se observan varias manchas de color rojizo-violeta que corresponden a los triterpenoides y esteroides en general. En el análisis de las fracciones obtenidas por la cromatografía en columna, se observaron en el extracto etéreo las manchas rojas sugiriendo esto que hay presentes esteroles. Además, se observan en forma débil manchas de color ladrillo como positiva para alcaloides. Los alcaloides fueron algunos de los metabolitos secundarios encontrados en el carpintero (aunque en menor proporción). De acuerdo con Kouznetsov (2005), algunos compuestos que pertenecen a esta familia se utilizan como medicamentos tales como quinina, estreptonigrina, cripolepirina y morfina, que tienen efectos antipalúdicos, antitumorales, antihipertensivos, antibióticos, analgésicos, citotóxicos, anticancerígenos, entre otros. Estos aspectos probablemente se deben a la efectividad del carpintero en aliviar algunas enfermedades informadas por la población estudiada.

El carpintero reportó la presencia de flavonoides; 
Tabla 3. Pruebas fitoquímicas realizadas a la especie Selaginella willdenowii

\begin{tabular}{llll}
\hline \multicolumn{1}{c}{ Metabolitos } & \multicolumn{3}{c}{ Pruebas realizadas } \\
& Éter de petróleo & Diclorometano & Acetato de etilo \\
\cline { 2 - 4 } Compuestos fenólicos & Negativo (-) & Negativo(-) & Negativo (-) \\
Taninos & Negativo (-) & Negativo (-) & Negativo (-) \\
Flavonoides & Positivo(+) & Positivo(+) & Positivo(+) \\
Triterpenoides y esteroides & Positivo(++) & Positivo(++) & Positivo(++) \\
Quinonas & Negativo (-) & Negativo (-) & Negativo (-) \\
Cardiotónicos & Negativo (-) & Negativo (-) & Negativo (-) \\
Alcaloides & Negativo (-) & Negativo (-) & Positivo(+) \\
\hline -: Ausente +: Presente en poca cantidad ++: Presente en mediana cantidad: +++: Presente en abundancia
\end{tabular}

esta familia de compuestos según Sánchez (1995), además de tener una acción antiinflamatoria, presentan actividad antiespasmódica, antimicrobial, anticancerígena, antitumoral, cardiovascular y en general proporcionan la materia prima para un gran número de drogas y aditivos; a su vez, son compuestos naturales que se encuentran en abundancia en las plantas, aparentemente tienen un papel funcional, ya que ayudan a la planta a reparar daños y la protegen de plagas y enfermedades. La gran mayoría de los flavonoides no son tóxicos para el hombre ni animales ya que se encuentran ampliamente distribuidos en los alimentos y son necesarios para obtener una buena salud. Según Anton (1988), Harbone (1967) y Zwi (1989), sólo algunos compuestos de este grupo son farmacológicamente activos y se les atribuye propiedades antiinflamatorias, antimicrobianas, analgésicas, de protección al sistema circulatorio e incluso como antivirales y anticancerígenos, lo que guarda mucha relación con lo que manifiesta la comunidad estudiada en cuanto a la categoría medicinal, cuando indican que la planta se utiliza para dolores en general (dolor de muelas, el dolor y la inflamación producida por mordedura de serpientes), para las contracciones intrauterinas durante el parto, etc.

De igual manera, se reportó la presencia de esteroides y/o triterpenoides, que son biomoléculas de gran importancia y pueden estar asociados con la actividad antiinflamatoria, específicamente actúan en enfermedades como la colitis ulcerativa y la enfermedad de Crohn, más conocida como inflamación intestinal, reduciendo de alguna manera la producción y actividad de citocinas (Arango y Mariño, 1998). Estos compuestos también actúan como anabólicos para el desarrollo de músculos, actividad citotóxica, inmunosupresora, actividad hipoglicemiante (disminuye el nivel de azúcar en la sangre) (Cárdenas, 2002). Asimismo, hay algunos compuestos esteroidales como la progesterona, típica hormona sexual femenina, que influye en el crecimiento, maduración, diferenciación y funcionamiento de las células nerviosas central (Gutiérrez y Contreras, 2000). Las hormonas esteroides se producen en células específicas de los testículos, la corteza adrenal, ovarios y placenta; sin embargo, juegan un papel a nivel fisiológico muy diferente, ya que están relacionadas con el embarazo, espermatogénesis, lactancia y parto, equilibrio mineral y metabolismo energético (aminoácidos, glúcidos y grasas).

Investigaciones realizadas por Silva et al. (1995) en Panamá, acerca de los compuestos químicos y la actividad biológica de la especie $S$. willdenowii, demuestran la presencia de flavonoides, por lo que fue considerada significativamente citotóxica al aplicársele a un grupo de humanos que presentaban algunas líneas celulares de cáncer. Estos aspectos fueron corroborados por Kinghorn et al. (1995), al obtener resultados similares en su estudio fitoquímico de esta especie. Relacionando este estudio y los anteriores cabe anotar que son una ayuda para validar el conocimiento tradicional que se tiene de esta especie en las comunidades encuestadas, porque se ratifica la presencia del mismo compuesto.

La información etnobotánica que se halló en esta investigación, es concordante con lo planteado por Litter (1978), quien en su trabajo farmacológico de esta especie presentó esteroides y alcaloides, los cuales consideró tenían acción oxitócica sobre el organismo de muje- 
res embarazadas; es decir, que estimula la motilidad uterina en forma selectiva, acelerando las contracciones sostenidas espasmódicas potentes. En este sentido, concluye que las sustancias oxitócicas en Sellaginella, ejercen acción directa sobre el miometrio, porque actúan sobre el útero aislado.

\section{Conclusiones}

Los resultados de esta investigación permitieron la determinación de las categorías de uso medicinal (54\%), mágico-religiosa (39\%) y ornamental (7\%) de la especie Sellaginella willdenowii, siendo los bebedizos, emplastos, perfumes y los baños de asiento las formas más prácticas de preparación.

Se puede considerar que el conocimiento etnobotánico sobre la especie debe estar estrechamente relacionado con la presencia de ciertos metabolitos secundarios como los flavonoides, esteroides, triterpenoides y alcaloides, de acuerdo con los reportes científicos sobre la actividad biológica que presentan estos compuestos.

La información obtenida constituye una fuente de conocimientos básicos que sirven para la domesticación, conservación y manejo de este recurso vegetal, que puede convertirse en fuente de importancia socioeconómica para la región, así como punto de partida para el desarrollo de otras investigaciones que permitan determinar con precisión los metabolitos secundarios que presenta ésta y otras especies florísticas.

\section{Literatura citada}

Adu-Tutu M, Elvin-Lewis M. 1980. Aproximación al análisis de datos en etnobotánica cuantitativa. Econ Bot. 33(3): 320-8.

Alston AHG, Jermy AC, Rankin JM. 1981. The genus selaginella in tropical South America. Bull Brit Mus (Nat Hist) Bot. 9: 233-303.

Alston AHG. 1955. The heterophyllousSelaginellae of continental North America.Bull Brit Mus (Nat Hist) Bot. 1: 221-74.

Arango H, Mariño G, Kestenberg A. 1998. Nuevos esteroides en enfermedad inflamatoria intestinal: uso de la Budesonida. Colomb Med. 29 (1): 24-25.

Balslev H, Forero E, Luteyn J. 1995. Biodiversity and conservation of neotropical montane forest. Vol 11. New York: The New York Botanical Garden. 390 p.

Bueno P, Palacios C. 2006. Etnobotánica de las especies útiles en la preparación de balsámicas en la región del San
Juan (Condoto, Opogodó y Nóvita (Chocó, Colombia). (Trabajo de grado). Quibdó: Universidad Tecnológica del Chocó «Diego Luis Córdoba». p. 32.

Caicedo M. 1983. Las oraciones y los secretos. En: Entorno al conocimiento tradicional. Quibdó: Instituto de Investigaciones Ambientales del Pacífico. $60 \mathrm{p}$.

Cárdenas D, Marín C, Suárez S, Guerrero C, Nofuya P. 2002. Plantas útiles en dos comunidades del departamento del Putumayo. Bogotá: Instituto Amazónico de Investigaciones Científicas, SINCHI. p. 67,125.

Cardona A. 1994. Algunas plantas utilizadas en la medicina tradicional por las mujeres de pie de pató Alto Baudó. Trabajo de grado. Universidad Tecnológica del Chocó «Diego Luis Córdoba», Quibdó, Chocó. 190 p.

Chan W, Hyun J, Han S, Duck H, Seop IH, Hyun CH, et al. 2008. Biflavonoids isolated from Selaginella tamariscina regulate the expression of matrix metalloproteinase in human skin fibroblasts. Bioorg Med Chem. 16 (2): 732-8.

Escobar RN, Rentería J, Vélez C, Rincón W, Ocampo E, Martínez Y, et al. 2003. Etnobotánica y comercialización de plantas aromáticas y medicinales en Quibdó, Chocó. Revista Institucional Universidad Tecnológica del Chocó. 19 (1): 12-8.

Forero E, Gentry A. 1989. Lista anotada de las plantas del departamento del Chocó, Colombia. Bogotá: Instituto de Ciencias Naturales (MHN), Universidad Nacional de Colombia.142 p.

Forero LE. 1980. Etnobotánica de las comunidades indígenas Cuna y Waunana, Chocó (Colombia). Cespedesia. 9 (3334): 114-306.

García-Cossio F, Echavarría J. 1999. La loca de sangre arriba. Medellín: Instituto Tecnológico Pascual Bravo. p. 27-30, 68.

Gallathri V, Asha A. 2005. Preliminary studies on the immunomodulatory and antioxidant properties of Selaginella species. Ind J Pharmacol. 37 (6): 381-5.

Gentry AH. 1986. Species richness and floristic compositation of Chocó regions plant communities. Caldasia. 15 (1): 71-91.

Gregory D, Riba R. 1979. Flora de Veracruz. Xalapa: Instituto Nacional de Investigaciones sobre Recursos Bióticos. p. $1-35$.

Gutiérrez AG, Contreras CM, Díaz JL. 2000. Cómo actúa la progesterona sobre el sistema nervioso central. Salud Mental. 23 (2): 42-8.

Holdridge LR. 1963. The determination of atmospheric water movements. Ecology. 43: 1-9.

Jermy AC. 1986. Subgeneric names in Selaginella. Fern Gaz. 13 (2):117-8.

Ke-Zheng X, Ke-Li K, Zhi-Wang Y, Sheng-Feng W. 2008. A new dihydrobenzofuran lignanoside from Selaginella moellendorffii Hieron. Chin Chem Lett. 19 (1): 79-81.

Kinghorn D. 1995. Estudio químico realizado sobre $S$. willdenowii. Maturitas. 22: 1-2.

Kouzetsov V. 2005. Recent progress in the synthesis of quinonines. Corren Org Chem 9 (1): 141-61.

Ling-Yun Ma, Shuang-Cheng Ma, Feng Wei, Rui-Chao Lin, Paul Pui-Hay But, Spencer Hon-Sun Lee, et al. 2007. A new sesquilignan from Selaginella sinensis (Desv.) Spring. Chin 
Chem Lett. 18 (10): 1224-6.

Litter M. 1978. Compendio de farmacología. Buenos Aires: Editorial El Ateneo. p 423-76.

Mahecha G. 1997. Fundamentos y metodología para la identificación de plantas. Bogotá: Proyecto Biopacífico, Miambiente -PNUD-GEF. p. 230.

Ma LY, Ma SC, Wei F, Lin RC, But PP, Lee SH, et al. 2003. Uncinoside and B, Two New Antiviral Chromone Glycosides from Selaginella uncinata. Chem Pharm Bull. 51(11): 1264-7.

Moreno D, Zapata K. 2008. Flora y fauna de uso medicinal en el municipio de Certegui, Chocó, Colombia. (Trabajo de grado). Quibdó: Universidad Tecnológica del Chocó «Diego Luis Córdoba». p. 31.

Mosquera M. 1996. Plantas medicinales de Tutunendo, Quibdó. Trabajo de grado. Universidad Tecnológica del Chocó «Diego Luis Córdoba», Quibdó, Chocó. p. 15.

Mosquera A, Rentería M. 2005. Caracterización de los huertos caseros (patios) en el corregimiento de Pacurita, Quibdó, Chocó, Colombia (Trabajo de grado). Quibdó: Universidad Tecnológica del Chocó «Diego Luis Córdoba». p. 43.

Murillo AJ. 2004. Catálogo de pteridofitos en el Chocó biogeográfico. En: Rangel O. (Ed.). Diversidad biótica IV: El Chocó biogeográfico/Costa pacífica. Bogotá: Unibiblos. p. 513-58.

Palacios L. 2008. Diversidad del género Selaginella P. Beauv (Selaginellaceae) en el departamento del Chocó. Bioetnia. 5 (2): 78-84.

Perechala A, Chaverra R, Rentería B, Hurtado L, Velásquez R, Echavarría J, et al. 1995. Entorno al conocimiento tradicional. Quibdó: Instituto de Investigaciones Ambientales del Pacífico. p. 34-80.

Plan de Ordenamiento Territorial municipio de Quibdó. 20012003. En línea [fecha de acceso: 25 de junio de 2008]. URL disponible en: http://www. alcaldidequibdo.gov.co.
Prada C. 2004. Helechos. En: Izco J. (Ed.). Botánica. $2^{\mathrm{a}}$ ed. Aravaca: McGraw-Hill Interamericana de España, S.A. p. 143-89.

Rangel CH, ArellanoPH. 2004. Clima del Chocó Biogeográfico de Colombia. En: Rangel O. (Ed.). Diversidad biótica IV: El Chocó biogeográfico/Costa pacífica. Bogotá: Unibiblos. p. 39-82.

Sáenz J, Noreña R. 2008 Manual de plantas naturales, fitoquímica de productos naturales. Madrid: Universidad de la República, Facultad de Química. p. 1-7.

Sanabria A. 1983. Análisis fitoquímico preliminar. Metodología $y$ aplicación en la evaluación de 40 plantas de la familia Compositae. Bogotá: Universidad Nacional de Colombia, Facultad de Ciencias, Departamento de Farmacia. p. 19.

Sánchez D. 1995. Métodos para evaluar la actividad biológica de los extractos (fundamentos teórico prácticos). Ibagué: Universidad del Tolima, Instituto de Ciencias Departamento de Biología. p. 17-20.

Silva GL, Chai H, Gupta MP, Farnsworth NR, Cordell GA, Pezzuto J, et al. 1995. Cytotoxic biflavonoids from Selaginella willdenowii. Phytochemistry. 40 (1): 129-34.

Sun CM, Syu WJ, Huang YT, Chen CC, Ou JC. 1997. Selective cytotoxicity of ginkgetin from Selaginella moellendorffii. J Nat Prod. 60 (4): 382-94.

Valdespino I. 1993. Flora of North America. Pteridophytes Gymnosperms. 45 (2): 315-27.

Yang SF, Chu SC, Liu SJ, Chen YC, Chang YZ, Hsieh YS. 2007. Antimetastatic activities of Selaginella tamariscina (Beauv.) on lung cancer cells in vitro and in vivo. $J$ Ethnopharmacol. 110 (3): 483-9.

Zheng X, Jiang D, Yunlong X, Bingyang Z, Duanfang L. 2007. A new steroid from Selaginella pulvinata. Fitoterapia 78 (2): 598-9. 